\title{
First, second, and third change of rings theorems for Gorenstein homological dimensions Driss Bennis and Najib Mahdou
}

\author{
Department of Mathematics, Faculty of Science and Technology of Fez, \\ Box 2202, University S. M. Ben Abdellah Fez, Morocco, \\ driss_bennis@hotmail.com \\ mahdou@hotmail.com
}

\begin{abstract}
In this paper, we investigate the change of rings theorems for the Gorenstein dimensions over arbitrary rings. Namely, by the use of the notion of strongly Gorenstein modules, we extend the well-known first, second, and third change of rings theorems for the classical projective and injective dimensions to the Gorenstein projective and injective dimensions, respectively. Each of the results established in this paper for the Gorenstein projective dimension is a generalization of a $G$-dimension of a finitely generated module $M$ over a noetherian ring $R$.
\end{abstract}

Key Words. Change of rings results; classical homological dimensions; Gorenstein homological dimensions; strongly Gorenstein projective and injective modules.

2000 Mathematics Subject Classification. 13D02; 13D05 ; 13 D07.

\section{Introduction}

All rings considered in this paper are assumed to be commutative with a unit; in particular, $R$ denotes such a ring. All modules are assumed to be unitary.

Setup and Notations. Let $M$ be an $R$-module. An element $x$ of $R$ is said to be $M$-regular, if $x \notin \mathcal{Z}_{R}(M)=\{r \in R \mid \exists m \in M-\{0\}, r m=0\}$ the set of zero-divisors on $M$. A sequence $x_{1}, \ldots, x_{n}$ of elements in $R$ is called an $M$-sequence, if $\left(x_{1}, \ldots, x_{n}\right) M \neq M$ and $x_{m} \notin \mathcal{Z}_{R}\left(M /\left(x_{1}, \ldots, x_{m-1}\right) M\right)$ for $m=1, \ldots, n$ (see for example [15] and [6]). For an element $x$ of $R$ which is neither a zero-divisor nor a unit, we use $\bar{R}$ to denote the quotient ring $R / x R$.

We use $\operatorname{pd}_{R}(M)$ and $\operatorname{id}_{R}(M)$ to denote, respectively, the classical projective and injective dimension of $M$.

We assume that the reader is familiar with the Gorenstein homological algebra (see references for a background. Namely, [6, 7, 9, 12, 14]).

The Gorenstein homological dimensions theory originated in the works of Auslander and Bridger 1] and [2], where they introduced the G-dimension of any finitely generated module $M$ and over 
any Noetherian ring $R$. The G-dimension is analogous to the G-dimension of a finitely generated module $M$ over a noetherian ring $R$ and shares some of its principal properties (see [6] for more details). However, to complete the analogy, an extension of the G-dimension to non-necessarily finitely generated modules is needed. This is done in the 1990's by Enochs and Jenda 10, 11, when they defined the Gorenstein projective dimension, as an extension of the G-dimension to modules that are not necessarily finitely generated and over arbitrary associative rings, and the Gorenstein injective dimension as a dual notion of the Gorenstein projective dimension:

Definition 1.1 - An $R$-module $M$ is called Gorenstein projective if there exists an exact sequence of projective $R$-modules,

$$
\mathbf{P}=\cdots \rightarrow P_{1} \rightarrow P_{0} \rightarrow P_{-1} \rightarrow P_{-2} \rightarrow \cdots,
$$

such that $M \cong \operatorname{Im}\left(P_{0} \rightarrow P_{-1}\right)$ and such that $\operatorname{Hom}_{R}(-, Q)$ leaves the sequence $\mathbf{P}$ exact whenever $Q$ is a projective $R$-module.

For a positive integer $n$, we say that $M$ has Gorenstein projective dimension at most $n$, and we write $\operatorname{Gpd}_{R}(M) \leq n$ (or simply $\operatorname{Gpd}(M) \leq n$ ), if there is an exact sequence of $R$-modules,

$$
0 \rightarrow G_{n} \rightarrow \cdots \rightarrow G_{0} \rightarrow M \rightarrow 0
$$

where each $G_{i}$ is Gorenstein projective.

- Dually, the Gorenstein injective module is defined, and so the Gorenstein injective dimension, $\operatorname{Gid}_{R}(M) \leq n$, of an $R$-module $M$ is defined.

The Gorenstein homological dimensions have been extensively studied by many others, who proved that these dimensions share many nice properties of the classical homological dimensions (see, for instance, 6, 9, 12]). In particular, several classical change of rings theorems have been extended to the Gorenstein homological dimensions setting (see, for example, 6], 8], and [17). However, most of those results are proved over Noetherian local rings, and in many cases the rings are in addition homomorphic images of Gorenstein local rings. In this paper, we generalize some change of rings results for Gorenstein homological dimensions by relaxing the conditions on the underlying rings. We are mainly interested in the Gorenstein counterpart of the classical first, second, and third change of rings theorems (see [15, Sections 4.4 and 4.5] and [19, Section 4.3]11). Namely, we extend these classical theorems to the Gorenstein setting.

Before investigating the first one, we establish, in Section 3, the second change of rings theorems for the Gorenstein projective and injective dimensions. Those are Theorems 3.1 and 3.4 .

Second change of rings theorems for the Gorenstein projective and injective dimensions.

Let $M$ be a non-zero $R$-module and let $x \in R$ be both $R$-regular and $M$-regular. Then,

1. $\operatorname{Gpd}_{\bar{R}}(M / x M) \leq \operatorname{Gpd}_{R}(M)$.

2. $\operatorname{Gid}_{\bar{R}}(M / x M) \leq \operatorname{Gid}_{R}(M)-1$, except when $M$ is a Gorenstein injective $R$-module.

These inequalities are extensions of the classical second change of rings theorems for the projective and injective dimensions ([19, Theorem 4.3.5] and [15. Theorem 205]), respectively. The first one is

\footnotetext{
${ }^{1}$ In this paper, we refer to Weibel's book [19] for the projective case and to Kaplansky's book [15] for the injective case.
} 
First, second, and third change of rings theorems for Gorenstein homological dimensions 3

a generalization of [6, Proposition 1.3.6], and the second one generalizes [16, Corollary 2.3] in which $R$ is assumed to be Noetherian local and $M$ to be finitely generated.

Section 4 is devoted to the first change of rings theorems the Gorenstein projective and injective dimensions. Recall the first change of rings theorem for the projective and injective dimensions ([19, Theorem 4.3.3] and [15, Theorem 202]):

First change of rings theorems for the projective and injective dimensions.

Let $M$ be a non-zero $R$-module and let $x=x_{1}, \ldots, x_{t}$ be an $R$-sequence of elements in the annihilator of $M$. Then,

1. If $\operatorname{pd}_{R /(x)}(M)<\infty$, then $\operatorname{pd}_{R}(M)=\operatorname{pd}_{R /(x)}(M)+t$.

2. If $\operatorname{id}_{R /(x)}(M)<\infty$, then $\operatorname{id}_{R}(M)=\operatorname{id}_{R /(x)}(M)+t$.

The first equality was extended for finitely generated modules and over Noetherian local rings to the Gorenstein projective dimension, as follows [6, Proposition 1.5.3]:

Let $R$ be a Noetherian local ring. Let $M$ be a non-zero $R$-module and let $x=x_{1}, \ldots, x_{t}$ be an $R$ sequence of elements in the annihilator of $M$. Then,

If $\operatorname{Gpd}_{R /(x)}(M)<\infty$, then $\operatorname{Gpd}_{R}(M)=\operatorname{Gpd}_{R /(x)}(M)+t$.

It is known that the first change of rings theorem for the projective dimension does not hold if we remove the condition $\operatorname{pd}_{R /(x)}(M)<\infty$. For example, we have $\operatorname{pd}_{\mathbb{Z} / 4 \mathbb{Z}}(\mathbb{Z} / 2 \mathbb{Z})=\infty$ but $\operatorname{pd}_{\mathbb{Z}}(\mathbb{Z} / 2 \mathbb{Z})=1$ [19, Example 4.3.1]. In this example, $\mathbb{Z} / 2 \mathbb{Z} \cong 2 \mathbb{Z} / 4 \mathbb{Z}$ is a Gorenstein projective $\mathbb{Z} / 4 \mathbb{Z}$-module. This implies that

$$
\operatorname{Gpd}_{\mathbb{Z}}(\mathbb{Z} / 2 \mathbb{Z})=\operatorname{Gpd}_{\mathbb{Z} / 4 \mathbb{Z}}(\mathbb{Z} / 2 \mathbb{Z})+1
$$

Similar example leads Christensen to ask whether the condition $\operatorname{Gpd}_{R /(x)}(M)<\infty$ in [6, Proposition 1.5.3] is necessary [6. Remarks 1.5.4]. Later, in [6, Theorem 2.2.8], he proved that certainly the equality $\operatorname{Gpd}_{R}(M)=\operatorname{Gpd}_{R /(x)}(M)+t$ holds without assuming the condition $\operatorname{Gpd}_{R /(x)}(M)<\infty$. In section 4, we generalize 6, Theorem 2.2.8]. Namely, we show that the equality $\operatorname{Gpd}_{R}(M)=$ $\operatorname{Gpd}_{R /(x)}(M)+t$ holds over arbitrary rings and for non-necessarily finitely generated modules. Also, we establish its dual result for the Gorenstein injective dimension. Those are Theorems 4.1 and 4.2

First change of rings theorem for the Gorenstein projective and injective dimensions. Let $M$ be a non-zero $R$-module and let $x=x_{1}, \ldots, x_{t}$ be an $R$-sequence of elements in the annihilator of $M$. Then,

$$
\operatorname{Gpd}_{R}(M)=\operatorname{Gpd}_{R /(x)}(M)+t \quad \text { and } \quad \operatorname{Gid}_{R}(M)=\operatorname{Gid}_{R /(x)}(M)+t .
$$

Finally, in Section 5, we discuss the third change of rings theorem.

In the next section, we give some definitions and results needed in the rest of the paper. 


\section{Preliminaries}

The proofs of almost all theorems given in the present paper are mainly based on the notion of "strongly Gorenstein projective and injective modules". These modules are introduced in [3], as particular cases of the Gorenstein projective and injective modules, respectively, as follows:

Definition $2.1([3]) \quad$ 1. An $R$-module $M$ is said to be strongly Gorenstein projective, if there exists an exact sequence of the form

$$
\mathbf{P}=\cdots \stackrel{f}{\longrightarrow} P \stackrel{f}{\longrightarrow} P \stackrel{f}{\longrightarrow} P \stackrel{f}{\longrightarrow} \cdots,
$$

where $P$ is a projective $R$-module and $f$ is an endomorphism of $P$, such that $M \cong \operatorname{Im}(f)$ and such that $\operatorname{Hom}_{R}(-, Q)$ leaves the sequence $\mathbf{P}$ exact whenever $Q$ is a projective $R$-module.

2. Dually the strongly Gorenstein injective $R$-modules is defined.

These particular cases of the Gorenstein projective and injective modules have simpler characterizations:

Proposition 2.2 ([3], Proposition 2.9 and Remark 2.10 (2)) 1. An R-module $M$ is strongly Gorenstein projective if and only if there exists a short exact sequence of $R$-modules

$$
0 \rightarrow M \rightarrow P \rightarrow M \rightarrow 0
$$

where $P$ is a projective $R$-module, and $\operatorname{Ext}_{R}^{1}(M, Q)=0$ for any $R$-module $Q$ with finite projective dimension (or for any projective $R$-module $Q$ ).

2. An R-module $M$ is strongly Gorenstein injective if and only if there exists a short exact sequence of $R$-modules

$$
0 \rightarrow M \rightarrow I \rightarrow M \rightarrow 0,
$$

where $I$ is an injective $R$-module, and $\operatorname{Ext}_{R}^{1}(E, M)=0$ for any $R$-module $E$ with finite injective dimension (or for any injective $R$-module $E$ ).

The principal role of the strongly Gorenstein projective and injective modules is to give a simple characterization of the Gorenstein projective and injective modules, respectively, as follows:

Theorem 2.3 ([3], Theorem 2.7) An R-module is Gorenstein projective (resp., injective) if and only if it is a direct summand of a strongly Gorenstein projective (resp., injective) $R$-module.

We also need the following extensions of the well-known standard (in)equalities for the projective dimension (4, Corollary 2, p. 135]) to the Gorenstein projective dimension.

Lemma 2.4 Let $0 \rightarrow A \rightarrow B \rightarrow C \rightarrow 0$ be a short exact sequence of $R$-modules. Then,

1. $\operatorname{Gpd}(A) \leq \sup \{\operatorname{Gpd}(B), \operatorname{Gpd}(C)-1\}$ with equality if $\operatorname{Gpd}(B) \neq \operatorname{Gpd}(C)$.

2. $\operatorname{Gpd}(B) \leq \sup \{\operatorname{Gpd}(A), \operatorname{Gpd}(C)\}$ with equality if $\operatorname{Gpd}(C) \neq \operatorname{Gpd}(A)+1$.

3. $\operatorname{Gpd}(C) \leq \sup \{\operatorname{Gpd}(B), \operatorname{Gpd}(A)+1\}$ with equality if $\operatorname{Gpd}(B) \neq \operatorname{Gpd}(A)$.

Proof. Using [14, Theorems 2.20 and 2.24] the argument is analogous to the one of 4, Corollary 2, p. 135]. 
First, second, and third change of rings theorems for Gorenstein homological dimensions 5

Some of these (in)equalities are already proved in special cases (see [6, Corollary 1.2.9] and [14] Proposition 2.18]).

Dually, we can prove the Gorenstein injective version of Lemma 2.4 above.

Lemma 2.5 Let $0 \rightarrow A \rightarrow B \rightarrow C \rightarrow 0$ be a short exact sequence of $R$-modules. Then,

1. $\operatorname{Gid}(A) \leq \sup \{\operatorname{Gid}(B), \operatorname{Gid}(C)+1\}$ with equality if $\operatorname{Gid}(B) \neq \operatorname{Gid}(C)$.

2. $\operatorname{Gid}(B) \leq \sup \{\operatorname{Gid}(A), \operatorname{Gid}(C)\}$ with equality if $\operatorname{Gid}(A) \neq \operatorname{Gid}(C)+1$.

3. $\operatorname{Gid}(C) \leq \sup \{\operatorname{Gid}(B), \operatorname{Gid}(A)-1\}$ with equality if $\operatorname{Gid}(B) \neq \operatorname{Gid}(A)$.

\section{Second change of rings theorems for the Gorenstein projective and injective dimensions}

This section is devoted to the second change of rings theorems for the Gorenstein projective and injective dimensions. We begin with the Gorenstein projective case, which is an extension of the well-known "second change of rings theorem for the projective dimension" [19, Theorem 4.3.5] and it is a generalization of [6, Proposition 1.3.6].

Theorem 3.1 Let $M$ be a non-zero $R$-module and let $x \in R$ be both $R$-regular and $M$-regular. Then,

$$
\operatorname{Gpd}_{\bar{R}}(M / x M) \leq \operatorname{Gpd}_{R}(M) .
$$

Proof. Since $\operatorname{Tor}_{i}^{R}(M, \bar{R})=0$ for all $i>0$ (by [4, Examples (1), p. 102]), the inequality follows by the following general change of rings result.

Lemma 3.2 Let $R \rightarrow S$ be a ring homomorphism with $\operatorname{pd}_{R}(S)<\infty$ and let $M$ be a non-zero $R$-module. If $\operatorname{Tor}_{i}^{R}(M, S)=0$ for all $i>0$, then $\operatorname{Gpd}_{S}\left(M \otimes_{R} S\right) \leq \operatorname{Gpd}_{R}(M)$.

Proof. To prove the inequality, we may assume that $\operatorname{Gpd}_{R}(M)=m<\infty$. First assume that $M$ is a Gorenstein projective $R$-module. We prove that $M \otimes_{R} S$ is a Gorenstein projective $S$-module. For that, we may assume, by [14, Theorem 2.5] and Theorem 2.3. that $M$ is strongly Gorenstein projective. Then, from Proposition 2.2 there exists a short exact sequence of $R$-modules

$$
0 \rightarrow M \rightarrow P \rightarrow M \rightarrow 0
$$

where $P$ is projective, and $\operatorname{Ext}_{R}^{1}(M, Q)=0$ for any $R$-module $Q$ with finite projective dimension. By the short exact sequence above, $\operatorname{Tor}_{i}^{R}(M, S) \cong \operatorname{Tor}_{i+1}^{R}(M, S)$ for all $i>0$. Then, $\operatorname{Tor}_{i}^{R}(M, S)=0$ for all $i>0\left(\right.$ since $\left.\operatorname{pd}_{R}(S)<\infty\right)$, and so we get the following exact sequence of $S$-modules

$$
0 \rightarrow M \otimes_{R} S \rightarrow P \otimes_{R} S \rightarrow M \otimes_{R} S \rightarrow 0 .
$$

On the other hand, consider a projective $S$-module $Q$. Then, $\operatorname{pd}_{R}(Q)$ is finite (by [5, Exercise 5 , p. 360]), and so, by [5. Proposition 4.1.3] and the fact that $M$ is a Gorenstein projective $R$-module

$$
\operatorname{Ext}_{S}^{1}\left(M \otimes_{R} S, Q\right) \cong \operatorname{Ext}_{R}^{1}(M, Q)=0 .
$$

Therefore, $M \otimes_{R} S$ is a strongly Gorenstein projective $S$-module (by Proposition 2.2).

Assume now that $m=\operatorname{Gpd}_{R}(M)>0$. Then, there exists a short exact sequence of $R$-modules,

$$
0 \rightarrow K \rightarrow F \rightarrow M \rightarrow 0
$$


where $F$ is free and, by Lemma $2.4 \operatorname{Gpd}_{R}(K)=m-1$. This, implies, by the induction hypothesis, that $\operatorname{Gpd}_{S}\left(K \otimes_{R} S\right) \leq m-1$. On the other hand, since $\operatorname{Tor}_{1}^{R}(M, S)=0$, we get a short exact sequence of $S$-modules

$$
0 \rightarrow K \otimes_{R} S \rightarrow F \otimes_{R} S \rightarrow M \otimes_{R} S \rightarrow 0 .
$$

Therefore, $\operatorname{Gpd}_{S}\left(M \otimes_{R} S\right)=\operatorname{Gpd}_{R}\left(K \otimes_{R} S\right)+1 \leq m$ (by Lemma 2.4 since $F \otimes_{R} S$ is a free $S$-module), which completes the proof.

Dually we prove the following general change of rings result, which is a generalization of 15 , Theorem 204].

Lemma 3.3 Let $R \rightarrow S$ be a ring homomorphism with $\operatorname{pd}_{R}(S)<\infty$ and let $M$ be a non-zero $R$-module. If $\operatorname{Ext}_{R}^{i}(S, M)=0$ for all $i>0$, then $\operatorname{Gid}_{S}\left(\operatorname{Hom}_{R}(S, M)\right) \leq \operatorname{Gid}_{R}(M)$.

Now, we give an extension of the "second change of rings theorem for the injective dimension" [15, Theorem 205]. This theorem generalizes [16, Corollary 2.3], which is obtained for finitely generated modules and over Noetherian local rings.

Theorem 3.4 Let $M$ be a non-zero $R$-module and let $x \in R$ be both $R$-regular and $M$-regular. Then,

$$
\operatorname{Gid}_{\bar{R}}(M / x M) \leq \operatorname{Gid}_{R}(M)-1,
$$

except when $M$ is a Gorenstein injective $R$-module (in which case $x M=M$ ).

Proof. First note that if $M$ is a Gorenstein injective $R$-module, then it is divisible; i.e., $x M=M$ for every non-zero-divisor element $x$ of $R$ (since it is a quotient of an injective module).

Now, to prove the inequality, we may assume that $\operatorname{Gid}_{R}(M)=n$ with $1 \leq n<\infty$. Then, there exists a short exact sequence of $R$-modules,

$$
0 \rightarrow M \rightarrow E \rightarrow I \rightarrow 0
$$

where $E$ is injective and, by Lemma 2.5, $\operatorname{Gid}_{R}(I)=n-1$. From [4, Examples (1), p. 102], we have that $\operatorname{Tor}_{1}^{R}(N, \bar{R})=N_{x}$ for all $R$-modules $N$, where $N_{x}$ denotes the submodule of $N$ annihilated by $x$. Then, Tensorising the short sequence above by $\bar{R}$ we get the following short exact sequence of $\bar{R}$-modules

$$
0 \rightarrow E_{x} \rightarrow I_{x} \rightarrow M / x M \rightarrow 0 .
$$

By [15, Theorem 204], $E_{x}$ is an injective $\bar{R}$-module, then the short exact sequence splits and so $M / x M$ is a direct summand of $I_{x}$. Thus, $\operatorname{Gid}_{\bar{R}}(M / x M) \leq \operatorname{Gid}_{\bar{R}}\left(I_{x}\right)$ (by Lemma 2.5). Finally, the desired inequality $\operatorname{Gid}_{\bar{R}}(M / x M) \leq n-1$ is obtained by the isomorphism $\operatorname{Hom}_{R}(\bar{R}, I) \cong I_{x}$ and by Lemma 3.3 (In fact, from [4, Examples (1), p. 102] and since $I$ is divisible as a quotient of the injective (then divisible) $R$-module $E, I$ satisfies the hypothesis $\operatorname{Ext}_{R}^{i}(\bar{R}, I)=0$ for all $i>0$ of Lemma 3.3.

\section{First change of rings theorems for the Gorenstein pro- jective and injective dimensions}

In this section, we investigate the first change of rings theorems for the Gorenstein projective and injective dimensions. We begin with the Gorenstein projective case.

As mentioned in the introduction, the classical first change of rings theorem for the projective 
dimension was already generalized and extended to the Gorenstein projective dimension for finitely generated modules and over Noetherian rings [6, Theorem 2.2.8]. Here, we show, by completely different arguments, that [6, Theorem 2.2.8] can be generalized to arbitrary rings and to nonnecessarily finitely generated modules. That is the following first change of rings theorem for the Gorenstein projective dimension.

Theorem 4.1 Let $M$ be a non-zero $R$-module and let $x=x_{1}, \ldots, x_{t}$ be an $R$-sequence of elements in the annihilator of $M$. Then

$$
\operatorname{Gpd}_{R}(M)=\operatorname{Gpd}_{R /(x)}(M)+t .
$$

In particular, $\operatorname{Gpd}_{R}(M)$ and $\operatorname{Gpd}_{R /(x)}(M)$ are simultaneously finite.

Proof. By induction on the length $t$ of the $R$-sequence $x$, it suffices to prove the case $t=1$, such that we write $x_{1}=x$ and $\bar{R}=R / x R$.

First we prove that $\operatorname{Gpd}_{\bar{R}}(M)<\infty$ implies $\operatorname{Gpd}_{R}(M)=\operatorname{Gpd}_{\bar{R}}(M)+1$. This gives the first inequality $\operatorname{Gpd}_{R}(M) \leq \operatorname{Gpd}_{\bar{R}}(M)+1$.

Assume then that $\operatorname{Gpd}_{\bar{R}}(M)=n<\infty$. We prove the equality $\operatorname{Gpd}_{R}(M)=n+1$ by induction on $n$. Assume first that $M$ is a Gorenstein projective $\bar{R}$-module and prove that $\operatorname{Gpd}_{R}(M)=1$. Note that $M$ can not be a Gorenstein projective $R$-module. Indeed, any Gorenstein projective $R$-module can be embedded in a free $R$-module and therefore can not have the $R$-regular element $x$ as a zero-divisor. So, it suffices to prove $\operatorname{Gpd}_{R}(M) \leq 1$. By Theorem [2.3 and 14, Proposition 2.19], we may consider $M$ to be a strongly Gorenstein projective $\bar{R}$-module. Thus, from Proposition 2.2 there exists a short exact sequence of $\bar{R}$-modules

$$
0 \rightarrow M \rightarrow P \rightarrow M \rightarrow 0
$$

where $P$ is a projective $\bar{R}$-module, and $\operatorname{Ext}_{\bar{R}}(M, Q)=0$ for any projective $\bar{R}$-module $Q$. From [18, Lemma 6.20], there is a commutative diagram:

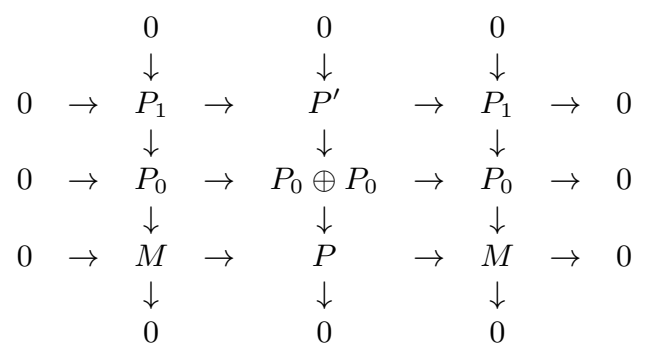

where $P_{0}$ is a projective $R$-module. Then, $P^{\prime}$ is a projective $R$-module (by [19, Theorem 4.3.3]). On the other hand, by the first vertical sequence in the diagram above, we have the isomorphism

$$
\operatorname{Ext}_{R}^{1}\left(P_{1}, Q\right) \cong \operatorname{Ext}_{R}^{2}(M, Q)
$$

for every $R$-module $Q$. If such an $R$-module $Q$ is projective (and so $Q / x Q$ is a projective $\bar{R}$-module), then the Rees's theorem [18, Theorem 9.37] gives

$$
\operatorname{Ext}_{R}^{2}(M, Q) \cong \operatorname{Ext}_{\bar{R}}^{1}(M, Q / x Q)=0 .
$$

Then, $\operatorname{Ext}_{R}^{1}\left(P_{1}, Q\right)=0$ and so $P_{1}$ is a strongly Gorenstein projective $R$-module (by Proposition 2.2). This means, by the diagram above, that $\operatorname{Gpd}_{R}(M) \leq 1$. Thus, the proof of the case $n=0$ is 
finished.

Now, assume that $\operatorname{Gpd}_{\bar{R}}(M)=n>0$. Then, there exists a short exact sequence of $\bar{R}$-modules,

$$
0 \rightarrow K \rightarrow F \rightarrow M \rightarrow 0
$$

where $F$ is free and, by Lemma $2.4 \operatorname{Gpd}_{\bar{R}}(K)=n-1$. Hence, by induction, $\operatorname{Gpd}_{R}(K)=n$. Therefore, $\operatorname{Gpd}_{R}(M)=\operatorname{Gpd}_{R}(K)+1=n+1\left(\right.$ by Lemma 2.4 and since $\operatorname{Gpd}_{R}(F)=\operatorname{pd}_{R}(F)=1$ (by [19, Theorem 4.3.3] and [14, Proposition 2.27])). This completes the proof of the first part.

Now, it remains to prove the inequality $\operatorname{Gpd}_{\bar{R}}(M)+1 \leq \operatorname{Gpd}_{R}(M)$. For that, we may assume that $\operatorname{Gpd}_{R}(M)=n<\infty$. As shown in the first part of this proof, $M$ can not be a Gorenstein projective $R$-module, and so we begin with the case $\operatorname{Gpd}_{R}(M)=1$ and we prove that $M$ is a Gorenstein projective $\bar{R}$-module. This is equivalent, by [14, Proposition 2.3], to show the following two statements:

1. $\operatorname{Ext}_{\bar{R}}^{i}(M, Q)=0$ for every $i \geq 1$ and every projective $\bar{R}$-module $Q$.

2. There exists an exact sequence of $\bar{R}$-modules

$$
\mathbf{P}=0 \rightarrow M \rightarrow P^{0} \rightarrow P^{1} \rightarrow \cdots
$$

such that $\operatorname{Hom}_{R}(-, Q)$ leaves the sequence $P$ exact whenever $Q$ is a projective $\bar{R}$-module.

To prove the first assertion, it suffices to consider $Q$ to be a free $\bar{R}$-module. In this case $Q$ is of the form $F / x F$, where $F$ is a free $R$-module. Therefore, from Rees's theorem [18, Theorem 9.37] and since $\operatorname{Gpd}_{R}(M)=1$, we get for every $i \geq 1$

$$
(*) \operatorname{Ext}_{\bar{R}}^{i}(M, F / x F) \cong \operatorname{Ext}_{R}^{i+1}(M, F)=0
$$

Now, we prove the existence of the resolution $\mathbf{P}$. For that, it suffices to prove the existence of short exact sequences

$$
0 \rightarrow M_{j} \rightarrow P^{j} \rightarrow M_{j+1} \rightarrow 0 \quad j \geq 0
$$

where $M_{0}=M$ and each $P^{j}$ is a projective $\bar{R}$-module, such that $\operatorname{Ext}_{\bar{R}}\left(M_{j}, Q\right)=0$ for any $j \geq 0$ and any projective $\bar{R}$-module $Q$.

Since $\operatorname{Gpd}_{R}(M)=1$, there exists a short exact sequence of $R$-modules

$$
0 \rightarrow G_{1} \rightarrow G_{0} \rightarrow M \rightarrow 0
$$

where $G_{0}$ is free and $G_{1}$ is Gorenstein projective. Tensorising this sequence by $\bar{R}$, we get the following exact sequence of $\bar{R}$-module:

$$
\left.\operatorname{Tor}_{1}^{R}\left(\bar{R}, G_{0}\right)=\right) 0 \rightarrow M \rightarrow G_{1} / x G_{1} \rightarrow G_{0} / x G_{0} \rightarrow M \rightarrow 0 .
$$

Setting $N=\operatorname{Ker}\left(G_{0} / x G_{0} \rightarrow M\right)$ we get two short exact sequences of $\bar{R}$-modules:

$$
0 \rightarrow M \rightarrow G_{1} / x G_{1} \rightarrow N \rightarrow 0 \quad \text { and } \quad 0 \rightarrow N \rightarrow G_{0} / x G_{0} \rightarrow M \rightarrow 0 .
$$

Since $G_{1}$ is a Gorenstein projective $R$-module, $x$ is $G_{1}$-regular. Then, by Theorem $3.1 G_{1} / x G_{1}$ is a Gorenstein projective $\bar{R}$-module. Thus, there exists, by definition, a short exact sequence of $\bar{R}$-modules

$$
0 \rightarrow G_{1} / x G_{1} \rightarrow P^{0} \rightarrow H_{0} \rightarrow 0
$$


First, second, and third change of rings theorems for Gorenstein homological dimensions 9

where $P^{0}$ is projective and $H_{0}$ is Gorenstein projective. Consider the following pushout diagram:

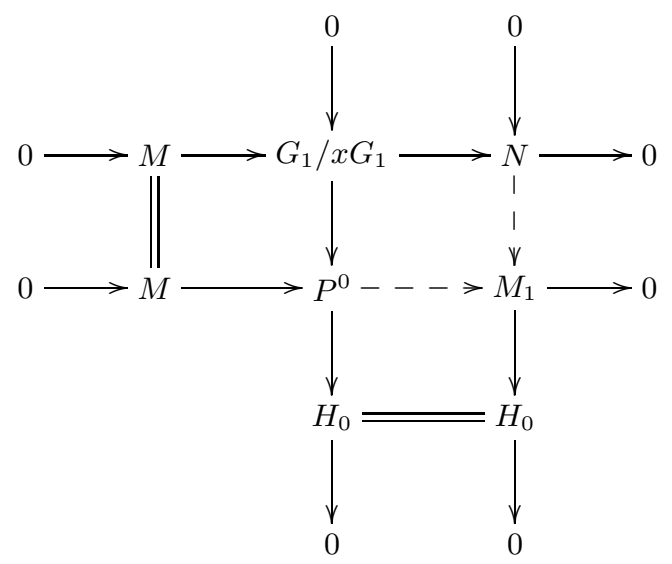

Since $G_{0}$ is a free $R$-module, $G_{0} / x G_{0}$ is a free $\bar{R}$-module, and so $\operatorname{pd}_{R}\left(G_{0} / x G_{0}\right)=1$. Then, $\operatorname{Gpd}_{R}(N) \leq 1$ (by Lemma 2.4 and by the short exact sequence of $\bar{R}$-modules $0 \rightarrow N \rightarrow G_{0} / x G_{0} \rightarrow$ $M \rightarrow 0)$. Then, using the same argument as above, we get that $\operatorname{Ext}_{\bar{R}}^{i}(N, Q)=0$ for every $i \geq 1$ and every projective $\bar{R}$-module $Q$. Thus, the middle horizontal sequence of the diagram above,

$$
0 \rightarrow M_{0}(=M) \rightarrow P^{0} \rightarrow M_{1} \rightarrow 0,
$$

is the desired first short exact sequence.

Now using the short exact sequence $0 \rightarrow N \rightarrow G_{0} / x G_{0} \rightarrow M \rightarrow 0$ and the right vertical sequence in the diagram above, we get the following pushout diagram:

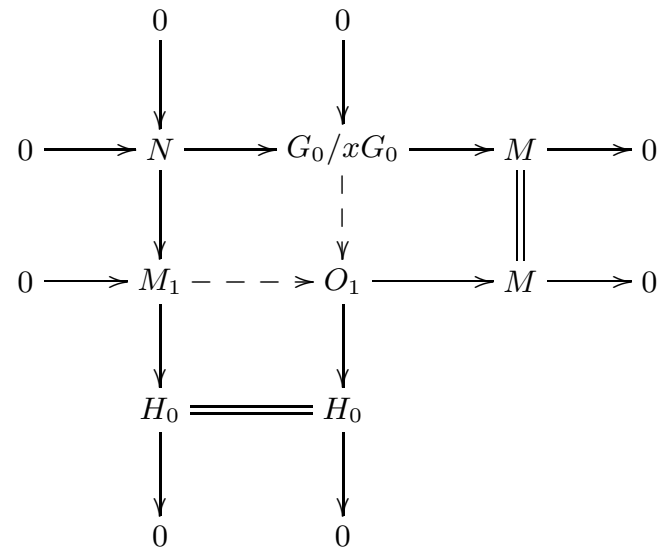

Since $O_{1}$ is a Gorenstein projective $\bar{R}$-module (apply [14, Theorem 2.5] to the middle vertical sequence in the diagram above), there exists a short exact sequence of $\bar{R}$-modules

$$
0 \rightarrow O_{1} \rightarrow P^{1} \rightarrow H_{1} \rightarrow 0,
$$


where $P^{1}$ is projective and $H_{1}$ is Gorenstein projective. With this sequence and the middle horizontal sequence in the diagram above we get the following pushout diagram:

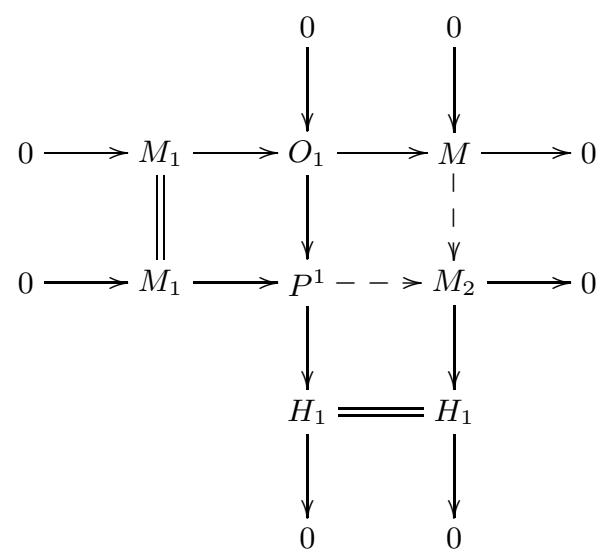

By the right vertical sequence, $\operatorname{Ext}_{\bar{R}}^{1}\left(M_{2}, Q\right)=0$ for any projective $\bar{R}$-module $Q$. Thus the desired second short exact sequence $0 \rightarrow M_{1} \rightarrow P^{1} \rightarrow M_{2} \rightarrow 0$ is established.

Recursively we construct the remains short exact sequences, and this completes the proof of the case $n=1$.

Finally, assume that $\operatorname{Gpd}_{R}(M)=n>1$ and consider a short exact sequence of $\bar{R}$-modules,

$$
0 \rightarrow K \rightarrow F \rightarrow M \rightarrow 0
$$

where $F$ is free. Since $\operatorname{pd}_{R}(F)=1$ and by Lemma 2.4 $\operatorname{Gpd}_{R}(K)=\operatorname{Gpd}_{R}(M)-1=n-1$. Thus, the induction hypothesis gives $\operatorname{Gpd}_{\bar{R}}(K) \leq \operatorname{Gpd}_{R}(K)-1=n-2$ and therefore

$$
\operatorname{Gpd}_{\bar{R}}(M)=\operatorname{Gpd}_{\bar{R}}(K)+1 \leq n-1
$$

This completes the proof.

Dually, we get the first change of rings theorem for the Gorenstein injective dimension.

Theorem 4.2 Let $M$ be a non-zero $R$-module and let $x=x_{1}, \ldots, x_{t}$ be an $R$-sequence of elements in the annihilator of $M$. Then,

$$
\operatorname{Gid}_{R}(M)=\operatorname{Gid}_{R /(x)}(M)+t .
$$

In particular, $\operatorname{Gid}_{R}(M)$ and $\operatorname{Gid}_{R /(x)}(M)$ are simultaneously finite.

\section{Third change of rings theorem for Gorenstein projec- tive dimension}

Recall the third change of rings theorem for the classical projective dimension [19, Theorem 4.3.12 and Remark p. 104]: Let $M$ be a non-zero $R$-module and let $x \in R$ be both $R$-regular and $M$ regular. If $R$ is Noetherian and $x$ is in the Jacobson radical of $R$, then $\operatorname{pd}_{\bar{R}}(M / x M)=\operatorname{pd}_{R}(M)$. 
First, second, and third change of rings theorems for Gorenstein homological dimensions 11

This theorem is extended to coherent rings and for finitely presented modules (see [13, Theorem 3.1.2]). In the Gorenstein dimensions theory, we find an extension of the third change of rings theorem for the projective dimension to the Gorenstein projective dimension of finitely generated modules and over Noetherian local rings (see [6, Corollary 1.4.6]). Here, as the classical case, we extend [6, Corollary 1.4.6] as follows:

Theorem 5.1 Let $R$ be a coherent ring and let $M$ be a non-zero finitely presented $R$-module. If $x=x_{1}, \ldots, x_{t}$ is an $R$-sequence in the Jacobson radical of $R$ and an $M$-sequence, then

$$
\operatorname{Gpd}_{R /(x)}(M /(x) M)=\operatorname{Gpd}_{R}(M) .
$$

Proof. Using [12, Proposition 10.2.6 $(1) \Leftrightarrow(10)$ ], the proof is the same as the one of [6, Corollary 1.4.6]. Here we only need to note that $R /(x)$ is also a coherent ring (by [13, Theorem 4.1 .1 (1)]) and over a coherent ring $R$ the $R$-module $\operatorname{Ext}_{R}^{n}(M, N)$ is coherent for every $n \geq 0$ and every coherent $R$-modules $M$ and $N$ (by 13 , Corollary 2.5.3]).

Finally, the authors have not been able to extend the third change of rings theorem for the classical injective dimension [15, Theorem 206] to the Gorenstein injective dimension over arbitrary Noetherian rings. However, there are some works which attempted to give such an extension. See, for instance, 16, Corollary 2.3] which shows that the desired extension holds over almost CohenMacaulay local rings.

Acknowledgements. The authors thank the referee for his/her careful reading of this work.

\section{References}

[1] Auslander, M. (1967). Anneaux de Gorenstein et torsion en algèbre commutative, Secrétariat mathématique, Paris. Séminaire d'algèbre commutative dirigé par Pierre Samuel, 1966/67. Texte rédigé, d'après des exposés de Maurice Auslander, par Marquerite Mangeney, Christian Peskine et Lucien Szpiro, Ecole Normale Superieure de Jeunes Filles.

[2] Auslander, M., Bridger, M. (1969). Stable module theory, Memoirs of the Amer. Math. Soc. 94.

[3] Bennis, D., Mahdou, N. (2007). Strongly Gorenstein projective, injective, and flat modules. J. Pure Appl. Algebra 210:437-445.

[4] Bourbaki, N. (1980). Algèbre Homologique, Chapitre 10. Masson, Paris.

[5] Cartan, H., Eilenberg, S. (1956). Homological Algebra. Princeton University Press, Princeton.

[6] Christensen, L. W. (2000). Gorenstein dimensions, Lecture Notes in Math. Berlin:SpringerVerlag.

[7] Christensen, L. W., Frankild, A., Holm, H. (2006). On Gorenstein projective, injective and flat dimensions - a functorial description with applications. J. Algebra 302:231-279.

[8] Christensen, L. W., Holm, H. (2009). Ascent properties of Auslander categories. Canad. J. Math. 61:76-108.

[9] Christensen, L. W., Foxby, H-B., Holm, H. (2009). Beyond Totally Reflexive Modules and Back. A Survey on Gorenstein Dimensions. To appear in Recent Developments in Commutative Algebra. Springer-Verlag. Available from arXiv:0812.3807v2. 
[10] Enochs, E. E., Jenda, O. M. G. (1993). On Gorenstein injective modules. Comm. Algebra 21:3489-3501.

[11] Enochs, E. E., Jenda, O. M. G. (1995). Gorenstein injective and projective modules. Math. Z. 220:611-633.

[12] Enochs, E. E., Jenda, O. M. G. (2000). Relative homological algebra. de Gruyter Expositions in Math. Berlin:Walter de Gruyter \& Co.

[13] Glaz, S. (1989). Commutative Coherent Rings. Lecture Notes in Math. Berlin:Springer-Verlag.

[14] Holm, H. (2004). Gorenstein homological dimensions. J. Pure Appl. Algebra 189:167-193.

[15] Kaplansky, I. (1970). Commutative Rings, Allyn and Bacon, Boston.

[16] Khatami, L., Yassemi, S. (2007). A Bass formula for Gorenstein injective dimension. Comm. Algebra 35:1882-1889.

[17] Khatami, L., Yassemi, S. (2003). Gorenstein injective and flat dimensions under base change. Comm. Algebra 31:991-1005.

[18] Rotman, J. (1979). An Introduction to Homological Algebra, Pure and Applied Math. New York-London:Academic Press.

[19] Weibel, C. A. (1994). An Introduction to Homological Algebra, Cambridge studies in advanced math. Cambridge:Cambridge University Press. 